\title{
Dynamic Renoscintigraphy in the Evaluation of Renal Function in Patients with Chronic Heart Failure
}

\author{
Zh V Vesnina* \\ Laboratory of Nuclear Medicine, Cardiology Research Institute, Tomsk National Research Medical Centre, Russian Academy of Sciences, \\ Russia \\ *Corresponding author: Zh V Vesnina, Laboratory of Nuclear Medicine, Cardiology Research Institute, Tomsk National Research \\ Medical Centre, Russian Academy of Sciences, Kievskaya str., 111a, 634012, Tomsk, Russian Federation
}

\begin{tabular}{lll}
\hline ARTICLE INFO & ABSTRACT \\
\cline { 1 - 1 } $\begin{array}{ll}\text { Received: } \\
\text { Published: June 18, } 2019\end{array} \quad \begin{array}{l}\text { Citation: Zh V Vesnina. Dynamic Renoscintigraphy in the Evaluation of Renal Function } \\
\text { in Patients with Chronic Heart Failure. Biomed J Sci \& Tech Res 19(1)-2019. BJSTR. }\end{array}$ \\
$\begin{array}{ll}\text { MS.ID.003250. } \\
\text { Keywords: Dynamic Renoscintigraphy; kidney Function; Heart Failure }\end{array}$ \\
\hline
\end{tabular}

\section{Introduction}

The relationship of cardiac and renal pathology has long attracted the attention of both cardiologists and nephrologists. In the past decade was noted that acute or chronic kidney damage is associated with high cardiovascular mortality [1]. On the other hand, in cardiovascular pathology an early assessment of renal damage is necessary for stratify the risk, develop strategies and management of such patients [2]. The aim of study is radionuclide estimation of the incidence and severity of renal dysfunction in patients with chronic heart failure (CHF).

\section{Materials and Methods}

The study included 235 patients (220 men and 15 women, mean age $56.24 \pm 7.35$ years) with a primary diagnosis: coronary heart disease complicated by CHF NYHA I-III; 169 (72\%) pts suffered one or more acute myocardial infarctions with the formation of postinfarction cardiosclerosis. All patients underwent dynamic radionuclide Renoscintigraphy with 99mTcdiethylenetriaminepentaacetic acid (99mTc-DTPA). The following parameters were calculated: total glomerular filtration rate (GFR, $\mathrm{mL} / \mathrm{min}$ ) and GFRs for each kidney; blood clearance (min) as half-time of radiopharmaceutical excretion from blood; halftime of radiotracer drainage from renal collecting system $\left(\mathrm{T}_{1 / 2}\right.$ (min), separately for the left and right kidneys); half-time of radiotracer drainage from renal parenchyma $\left(\mathrm{T}_{1 / 2 \mathrm{PAR}}\right.$, separately for the left and right kidneys). The scintigraphy studies were performed with gamma camera Philips Forte (Philips Medical
Systems, Netherlands). Processing of the acquired scintigrams was performed using JetStream ${ }^{\circledR}$ Workspace Release 3.0 software package (Philips Medical Systems, Netherlands).

\section{Results and Discussion}

According to the results of radionuclide Renoscintigraphy functional renal activity disorders were not identified in only 44 (18.7\%) out of 235 patients studied. However, only 57 (24.3\%) patients had chronic renal pathology in anamnesis, including: chronic pyelonephritis - in 32 patients, urolithiasis - in 15 patients, polycystic kidney disease - in 6 patients, chronic renal insufficiency - in 2 patients and 1 case of renal artery stenosis and nephropathy. Decreased glomerular filtration rate of one or both kidneys, in greater and lesser extent, was found in 177 (75.3\%) out of the 235 patients studied. This is also demonstrated in the studies of McAlister FA [3] which showed the presence of functional renal insufficiency in $56.6 \%$ of patients with CHF NYHA II-III. The mean GFR values were $105.13 \pm 8.36 \mathrm{~mL} / \mathrm{min}$ (total GFR); $47.60 \pm 6.57$ $\mathrm{mL} / \mathrm{min}$ for the left kidney and $57.52 \pm 6.01 \mathrm{~mL} / \mathrm{min}$ for the right kidney. In 49 (20.9\%) patients, we found pronounced renal dysfunction (the GFR decline in one or both kidneys by more than $30 \%$ of normal values).

The majority of patients (101 (43\%) patients) had moderately pronounced changes in the filtration function and manifested GFR decline by $15-30 \%$ of normal values. Minor disorders ( $<15 \%$ of normal values) were identified in 27 out of 235 (11.5\%) patients 
studied. It is known that at early stages of heart insufficiency a decrease in renal blood flow does not affect GFR or affects it slightly, then as the disease progresses there is a decrease in GFR due to the renal perfusion reduction and increase in renal vascular resistance [4]. But according to other authors, renal functional reserve may be reduced at the early stages of CHF (NYHA I) and even in cases of clinically asymptomatic left ventricular dysfunction [5]. Evacuation disorders in parenchyma were found in 93 patients (39.6\% out of the examined patients). The identified disorders were mostly moderate and minor (not more than 15 minutes over the normal range upper limit) (57 (24.3\%) and 18 (7.7\%) patients, respectively).

The exceptions were 18 patients who had a significantly delayed indicator clearance $\left(\mathrm{T}_{1 / 2 \mathrm{PAR}}\right.$ was more than $\left.35 \mathrm{~min}\right)$. The latter fact should be emphasized because it indicates that the indicator excretion disorder in these patients was due to changes in the renal parenchyma that was above the pelvicalyceal system level and indicated renal tubular reabsorption disorders. On the basis of this, the deviation of such indicators as $\mathrm{T}_{1 / 2 \text { PAR }}$ from the normal range can also be regarded as the result of damage to the central and renal hemodynamics in patients with coronary heart disease complicated with chronic CI $[6,7]$. It should be noted that the last two indicators show the activity of excretion processes occurring in the nephron. The delay in indicator excretion from pelvicalyceal system occurred in $65(27.7 \%)$ cases and mostly (45 patients) it was a small extent (no more than 6 minutes over the upper limit of normal range). The disorder of evacuation function was mild in 14 patients $(6.0 \%)$ and quite significantly pelvicalyceal activity was reduced in $6(2.6 \%)$ patients $\left(\mathrm{T}_{1 / 2}\right.$ was more than 30 minutes).

\section{Conclusion}

Thus, the results of radionuclide Renoscintigraphy suggest that renal dysfunction progresses in not less than $75 \%$ of patients with coronary artery disease complicated by chronic heart failure. Decreased contractility of the heart and the associated renal hypoperfusion determine a decrease in glomerular filtration rate, tubular reabsorption and impaired excretion processes in patients with chronic heart failure.

\section{Statement of Ethics}

The study protocol complied with the Declaration of Helsinki and was approved by the Ethics and Research Committee of the Cardiology Research Institute, Tomsk National Research Medical Centre. All patients have given their informed written consent.

\section{References}

1. Sarnak MJ (2003) Cardiovascular complications in chronic kidney disease. Am J Kidney Dis 41(5Supply): 11-17.

2. Dries DL, Exner DV, Domanski MJ (2000) The prognostic implications of renal insufficiency in asymptomatic and D.V.symptomatic patients with left ventricular systolic dysfunction. J Am Coll Cardiol 35: 681-689.

3. McAlister FA, Ezekowitz J, Tonelli M, Armstrong PW (2004) Renal Insufficiency and Heart Failure. Circulation 2004; 109: 1004-1009.

4. Ronco C, Ronco F (2012) Cardio-renal syndromes: a systematic approach for consensus definition and classification. Heart Fail Rev 17(2): 151160.

5. Magri P, Rao MAE, Cangianiello S, Bellizzi V, Rosario Russo, et al. (1998) Early Impairment of Renal Hemodynamic Reserve in Patients with Asymptomatic Heart Failure Is Restored by Angiotensin II Antagonism. Circulation 98: 2849-2854.

6. Eriksen R, Vegsundvaag J, Hole T, Morsto TH (2006) Hepatic and renal haemodynamics changes in congestive heart disease. J Tidsskr Nor Laegeforen 126(6): 743-746.

7. De Santo NG, Cirillo M, Perna A, Pollastro RM, Frangiosa A, et al. (2005) The kidney in heart failure. Semin J Nephrol 25(6): 404-407.

\section{ISSN: 2574-1241}

DOI: 10.26717/BJSTR.2019.19.003250

Zh V Vesnina. Biomed J Sci \& Tech Res

CC (P) This work is licensed under Creative

Submission Link: https://biomedres.us/submit-manuscript.php

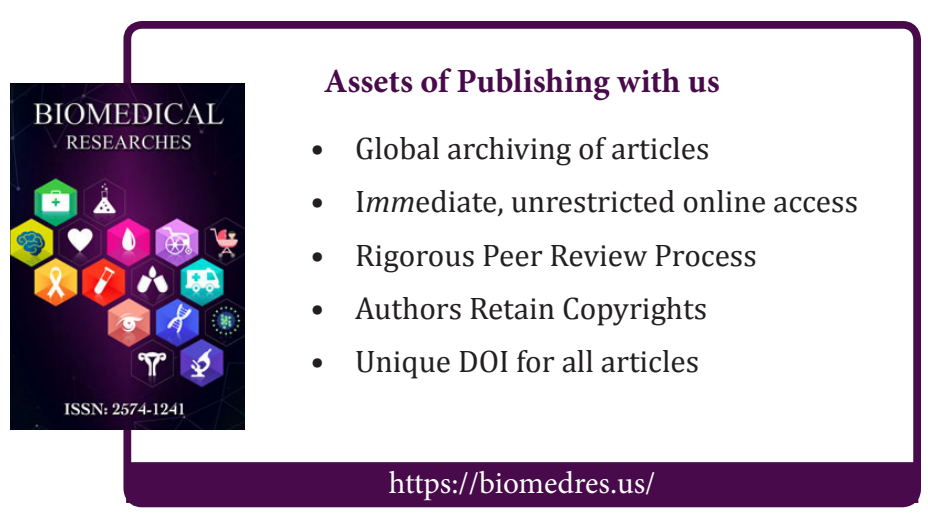

